\title{
COROIDECTOMÍA EN MELANOCITOMA COROIDEO. CARACTERÍSTICAS CLÍNICAS E HISTOPATOLÓGICAS
}

\author{
CHOROIDECTOMY IN CHOROIDAL MELANOCYTOMA. \\ CLINICAL AND HISTOPATHOLOGIC CHARACTERISTICS
}

\author{
PELAYES DE ${ }^{1}$, ZARATE JO ${ }^{2}$
}

\begin{abstract}
RESUMEN
Objetivo/método: Se presenta el caso de un tumor infrecuente, el melanocitoma de localización coroidea. Las características clínicas, y ecográficas son similares al melanoma coroideo. Se realizó como tratamiento una coroidectomía.

Se exponen las características histopatológicas utilizando hematoxilina y eosina, PAS, tricrómico de Masson e inmunohistoquímica.

Resultados/conclusiones: Se presentan las características clínicas e histopatológicas del melanocitoma coroideo, cuyas características clínicas son similares al melanoma coroideo y las histopatológicas iguales a los melanocitomas de otras partes del ojo. Es muy difícil diferenciar ambos tumores sin correlaciones histopatológicas.
\end{abstract}

Palabras claves: Melanocitoma, coroidectomía, resección local, coroides, inmunohistoquímica.

\begin{abstract}
Purpose/methods: To report a rare case of melanocytoma in a choroidal location. The clinical and echographic characteristics were similar to those of a melanoma. The treatment selected in our case was a choroidectomy.

Tissue was stained with hematoxylin-eosin, periodic acid-Schiff, Masson's trichromic and immunohistochemistry was performed.

Results/conclusions: The clinical and histopathologic features of choroidal melanocytoma are similar to the characteristics of a melanocytoma located in other parts of the eye. The differential diagnosis between choroidal melanoma and melanocytoma is very difficult without histopathologic correlation (Arch Soc Esp Oftalmol 2006; 81: 341-344).
\end{abstract}

Key words: Melanocytoma, choroidectomy, local resection, choroid, immunohistochemistry.

Recibido: 22/11/05. Aceptado: 16/6/06.

Universidad de Buenos Aires. Buenos Aires. Argentina.

1 Doctor en Medicina. Docente Autorizado Oftalmología. Hospital General de Agudos Dr. Carlos G. Durand. II Cátedra de Oftalmología.

Universidad de Buenos Aires. LIOCiV Laboratorio de Investigaciones Oftalmológicas y Ciencias Visuales, Facultad de Medicina.

2 Doctor en Medicina. Profesor Adjunto en Patología. Departamento de Patología. Universidad de Buenos Aires. LIOCiV Laboratorio de Investigaciones Oftalmológicas y Ciencias Visuales, Facultad de Medicina.

Correspondencia:

David E. Pelayes

Castro Barros 321

CP 1178

Buenos Aires

Argentina

E-mail: dpelayes@ciudad.com.ar 


\section{INTRODUCCIÓN}

Descrito por Zimmerman en 1962 (1) el melanocitoma uveal es una variante del nevus (nevus magnocelular); su localización clásica es en la papila o cerca de ésta, pero puede localizarse en cualquier sector del tracto uveal como cuerpo ciliar, iris y raramente en la coroides. Es un tumor benigno, congénito no hereditario e intensamente pigmentado que se presenta en individuos de color con mayor frecuencia y que raramente puede malignizarse. La agudeza visual está conservada, pero en un tercio de los casos se describió un defecto pupilar aferente.

La localización clásica de esta entidad no presenta en la mayoría de los casos un problema diagnóstico y terapéutico el cual habitualmente es el control clínico ecográfico anual y eventualmente fotografiar la lesión. Sin embargo no sabemos si podemos extrapolar las características antes mencionadas con la localización coroidea y esto dificulta su diagnostico de certeza en esta ubicación con el melanoma coroideo $(2,3)$.

Se presenta un caso de melanocitoma coroideo tratado con coroidectomía.

\section{CASO CLÍNICO}

Paciente de sexo femenino de 59 años de edad que es derivada para enucleación del ojo izquierdo (OI) con el diagnóstico de presunción de un melanoma de coroides ubicado a nivel temporal superior.

Sin antecedentes patológicos personales ni heredo familiares de importancia.

Agudeza visual ojo derecho (OD) 1 y OI bultos, el segmento anterior, sin particularidades, presión ocular: OD 15 y OI $23 \mathrm{mmHg}$. En el segmento posterior se evidencia un tumor hiperpigmentado, ubicado a nivel temporal superior, de coloración variable del naranja al marrón oscuro con zonas de hemorragia en su superficie, líquido subretiniano alrededor y leve hemovítreo (fig. 1).

Como exámenes complementarios se realizaron: retinofluoreceinografía (AFG) que muestra: neovasos sobre la superficie (fig. 2) y fuga de colorante en tiempos tardíos en la base de la lesión, ecografía: que mostró un tumor sólido, de 10,16 mm de altura y $6,25 \mathrm{~mm}$ de base, aspecto fúngico, reflectividad media baja y excavación coroidea (fig. 3).

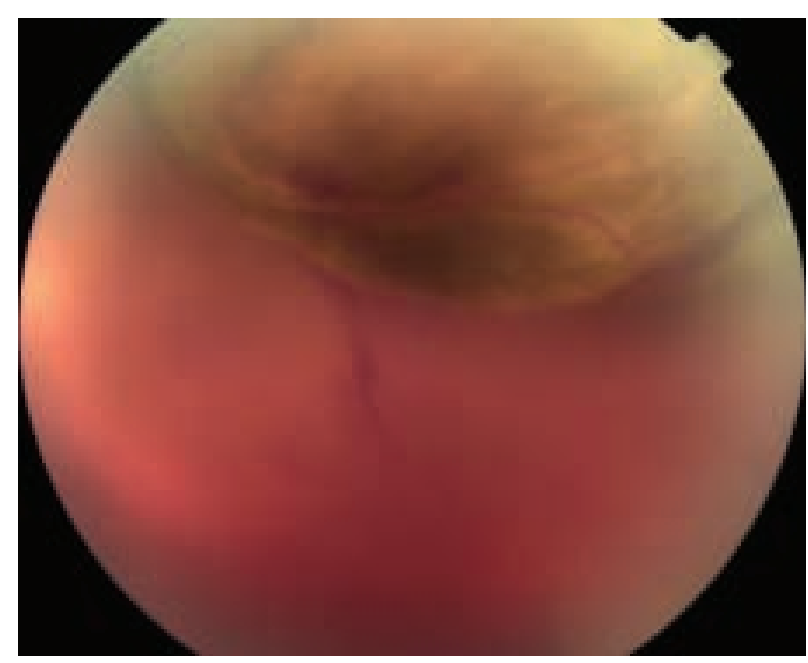

Fig. 1: Retinografía: tumor hiperpigmentado con zonas de hemorragia en su superficie.

Los exámenes clínicos y oncológicos resultaron negativos para descartar foco oncológico primario o matastásico.

Con los datos obtenidos se presumió el diagnóstico clínico-ecográfico de melanoma de coroides. El paciente prefirió un tratamiento conservador, realizándose coroidectomía (esclerouvectomía lamelar parcial) bajo anestesia general hipotensiva.

Presentó una buena evolución en el postoperatorio. No se observaron recidivas locales o sistémicas durante los 9 meses de seguimiento.

La pieza quirúrgica fue estudiada con Hematoxilina-Eosina, Tricromico de Masson, PAS e inmu-

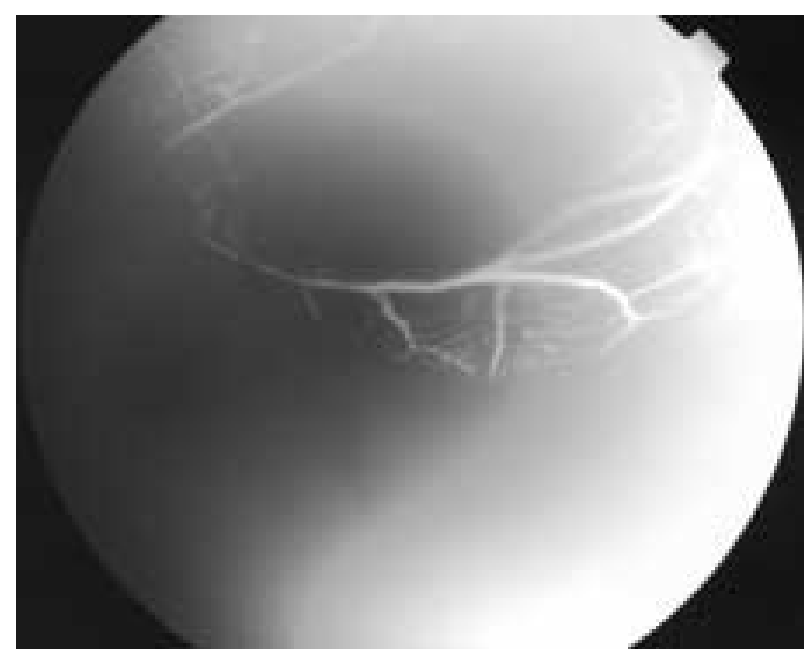

Fig. 2: Retinofluoresceinografía: se observan neovasos en la superficie del tumor. 


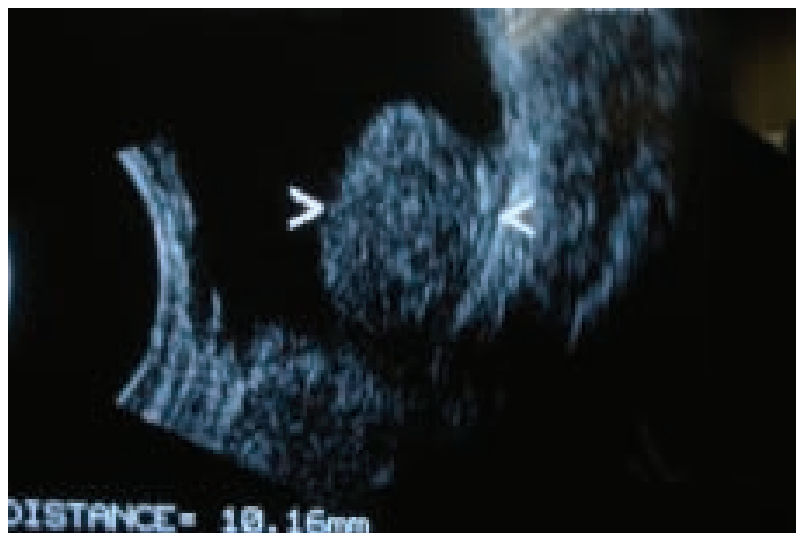

Fig. 3: Ecografia: tumor fúngico de reflectividad media baja.

nohistoquimica (proteína S-100, vimentina, MelanA y HMB45). El estudio histopatológico reveló la presencia de un tumor intensamente pigmentado con las características de los melanocitomas de coroides.

Los estudios inmunohistoquímicos fueron positivos para proteína S-100 y vimentina (fig. 4), y negativos para Melan-A y HMB45. Tras la pigmentación con agua oxigenada al 5\%, en diferentes cortes para poder aplicar la solución durante diferentes períodos $(24,48$ y $72 \mathrm{~h}$ ) se observaron nítidamente las clásicas células de citoplasma claro, con núcleos típicos y regulares de carácter névico, características fundamentales del melanocitoma (figs. 5 y 6).

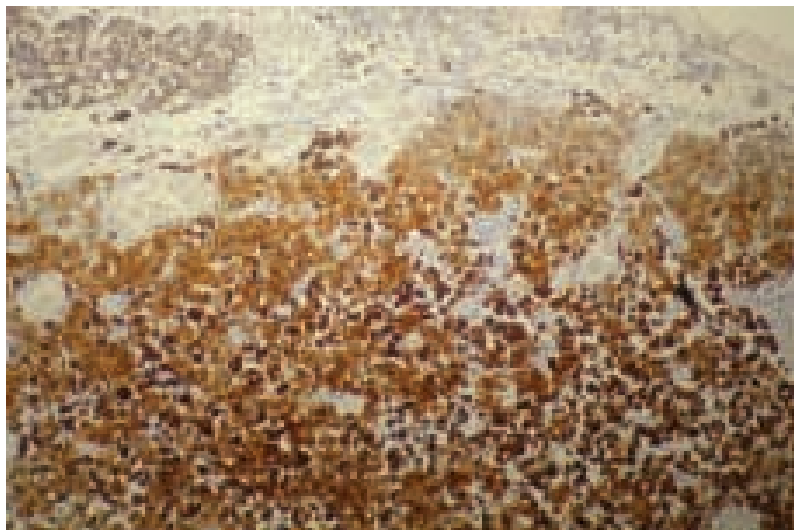

Fig. 4: Panorámica de inmunohistoquímica. Vimentina positiva.

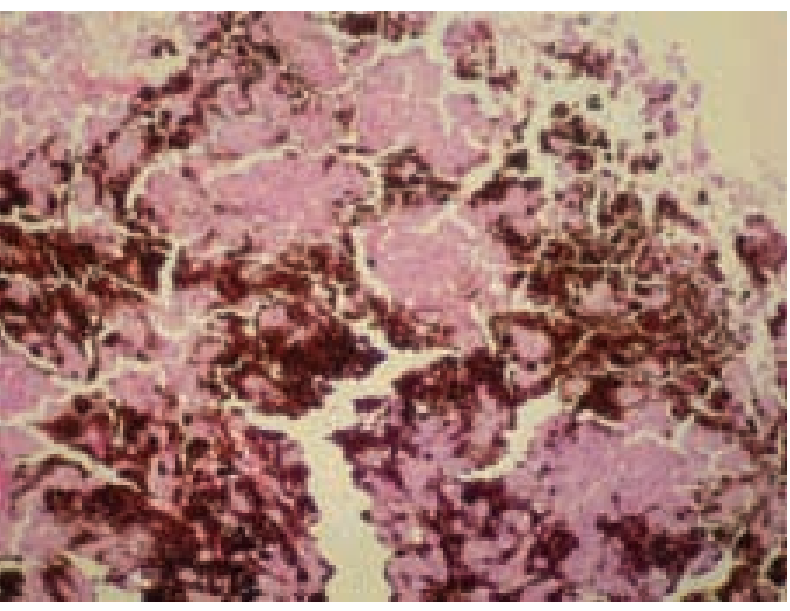

Fig. 5: Histopatología: Hematoxilina y Eosina con pigmentación.

\section{DISCUSIÓN}

La localización clásica de este tumor no presenta en la mayoría de los casos un problema diagnóstico o terapéutico, aquellos casos en los que se certifica crecimiento y disminución de la agudeza visual puede sugerir un cambio maligno (4). Sin embargo desconocemos si se podían extrapolar las características antes mencionadas con la localización coroidea. Por ser ésta la menos frecuentemente presentada no tenemos certeza de su comportamiento biológico.

En la bibliografía consultada (MedLine) no hemos encontrado ninguna característica singular

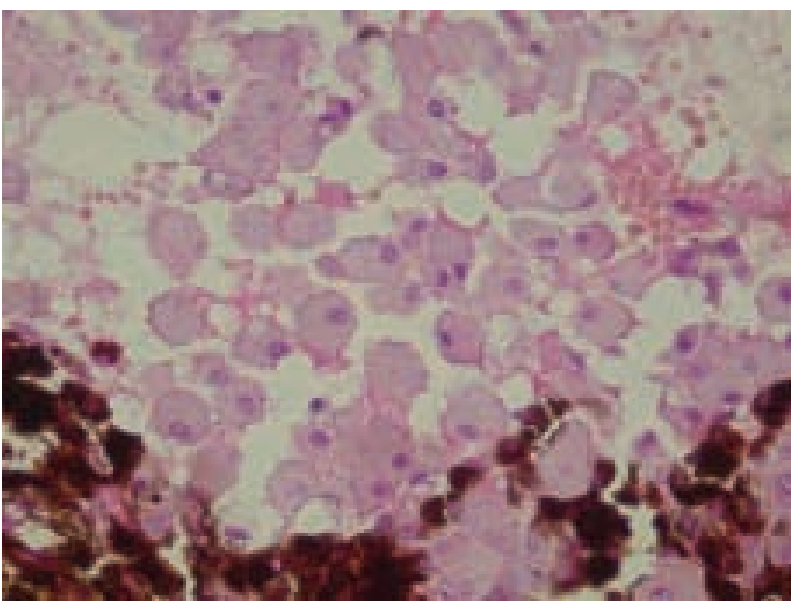

Fig. 6: Histopatología: Hematoxilina y Eosina con depigmentación a las 48 horas. 
tanto clínica, ecografía o en la AFG que pueda diferenciar claramente el melanoma coroideo del melanocitoma de igual localización. De hecho en el caso presentado, al igual que en artículos previos, las características ecograficas, clínicas son similares al melanoma y en la retinofluoresceinografía no se presenta el típico bloqueo descrito en las localizaciones papilares, debido a su ubicación en coroides.

En la mayoría de los casos publicados de melanocitoma coroideo el tratamiento fue la enucleación, salvo en uno que se realizó la reseccion local (5), siendo el único caso comunicado con esta técnica quirúrgica hasta la fecha, en que se comunica el segundo caso con este tratamiento.

En resumen, aunque es un tumor relativamente infrecuente, localizaciones extra papilares representan un desafío diagnóstico muy importante por su semejanza clínica y ecografía con el melanoma coroideo.

No se ha encontrado suficiente evidencia en la literatura consultada que permita ofrecer una pauta de tratamiento adecuado frente a la presencia del melanocitoma de coroides. Tal vez los tratamientos conservadores puedan ser una opción en casos dudosos o eventualmente tener diagnóstico citológico previo a tratamientos radicales (la punción aspirativa con aguja fina puede ser una alternativa a considerar).

Sólo nuevos casos con correlación clínico patológica permitirán conocer mejor esta entidad.

\section{BIBLIOGRAFÍA}

1. Zimmerman LE, Garron LK. Melanocytoma of the optic disc. Int Ophthalmol Clin 1962; 2: 431-440.

2. Shields JA, Font RL. Melanocytoma of the choroid clinically simulating a malignant melanoma. Arch Ophthalmol 1972; 87: 396-400.

3. Robertson DM, Campbell RJ, Salomao DR. Mushroomshaped choroidal melanocytoma mimicking malignant melanoma. Arch Ophthalmol 2002; 120: 82-85.

4. Shileds JA, Shields CL, Eagle RC Jr, Lieb W E, Stern S. Malignant melanoma associated with melanocytoma of the optic disc. Ophthalmology 1990; 97: 225-230.

5. Raichand M, Peyman GA, Juarez CP, Seetner AA, Sugar $J$, Goldberg MF. Resection of uveal melanocytoma: clinicopathological correlation. Br J Ophthalmol 1983; 67 : 236-243. 